\title{
Contaminación lumínica,
} DESAFÍO PARA LOS NUEVOS TIEMPOS

\section{LiGHT POLLUTION, CHALLENGE FOR THE NEW TIMES}

Ph.D. Millard Escalona Hernandez

Universidad Tecnológica Israel mescalona@uisrael.edu.ec

Fecha de recepción: 06/05/2015

Fecha de aceptación: 25/06/2015

\begin{abstract}
Resumen:
El crecimiento de la humanidad no sólo en cantidad de individuos, también en tecnología, expectativa de vida y usos de recursos se ha extendido por todo el planeta. Estos factores van de la mano con un crecimiento económico el cual ha sido liderado por los países desarrollados, generando un crecimiento de sus principales ciudades y por lo tanto un aumento demográfico de las misma, traduciéndose en mayor demanda de servicios en tal sentido utilizando la totalidad de las horas disponibles de un día, haciendo de la noche una alternativa para la producción laboral y de recreación.
\end{abstract}

En las últimas décadas, el grado y la intensidad de la iluminación artificial de la noche se han incrementado, generando efectos negativos sobre hábitats y especies, en particular en la salud humana.

Palabras clave: Contaminación Lumínica; efectos en la salud humana; Impacto sobre lo natural; Qué hacer. 


\section{Abstract:}

The growth of humanity not only in quantity of individuals, but also in technology, life expectancy and uses of resources has been spread throughout the planet. These factors go hand in hand with economic growth which has been led by developed countries, generating a growth of its major cities and therefore a population increase of the same, translating into greater demand for services in this regard using all the hours available for a day, making the night an alternative for the production work and recreation.

In the last few decades, the degree and intensity of the artificial lighting of the night has increased, generating negative effects on habitats and species, in particular on human health.

Keywords: Light pollution; effects on human health; impact on the natural; What To Do 


\section{Introducción}

Cualquier teoría física es siempre provisional, en el sentido de que es sólo una hipótesis nunca se puede probar. A pesar de que los resultados de los experimentos concuerden muchas veces con la teoría, nunca podremos estar seguros de que la próxima vez el resultado no vaya a contradecirla. Sin embargo, se puede rechazar una teoría en cuanto se encuentre una única observación que contradiga sus predicciones. Como ha subrayado el filósofo de la ciencia Karl Popper, "una buena teoría está caracterizada por el hecho de predecir un gran número de resultados que en principio pueden ser refutados o invalidados por la observación".

Cada vez que se comprueba que un nuevo experimento está de acuerdo con las predicciones, la teoría sobrevive y nuestra confianza en ella aumenta. Pero si por el contrario se realiza alguna vez una nueva observación

Figura 1: Cielo nocturno en Italia.

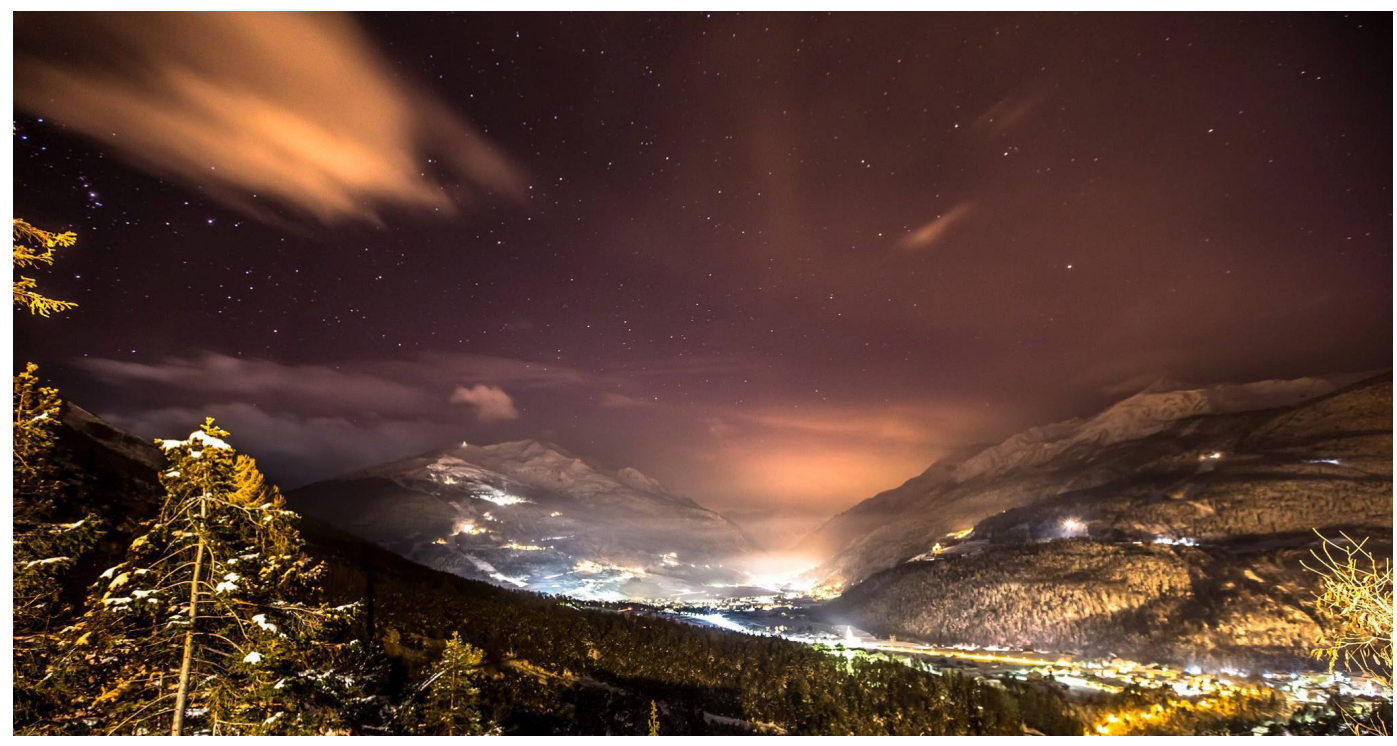

Fuente: Google earth. 2015 
que contradiga la teoría, tendremos que abandonarla o modificarla. $\mathrm{O}$ al menos esto es lo que se supone que debe suceder, aunque siempre puede cuestionar la competencia de la persona que realizó la observación.

En la práctica, sucede que se construye una nueva teoría que en realidad es una extensión de la teoría original. El objetivo final de la ciencia es el proporcionar una única postura que describa correctamente toda la visión. Sin embargo, el método que la mayoría de los científicos siguen en realidad es el de separar el problema en dos partes. Primero, están las leyes que nos dicen cómo cambia el universo con el tiempo. (Si conocemos cómo es el universo en un instante dado, estas leyes físicas nos dirán cómo será el universo en cualquier otro posterior.) Segundo, está la cuestión del estado inicial del universo, desde el origen de la civilización, el humano no se ha contentado con ver los acontecimientos como desconectados e inexplicables. Ha buscado incesantemente un conocimiento del orden subyacente del mundo, hoy en día, aún seguimos anhelando saber por qué estamos aquí y de dónde venimos. El profundo deseo de conocimiento de la humanidad es justificación suficiente para continuar nuestra búsqueda.

El objetivo principal de la presente investigación fue determinar los niveles de contaminación lumínica producto de la acción del hombre en el medio natural, en las especies y en la salud del ser humano. Y el abordaje de los factores de que incide en los niveles de contaminación.

\section{METODOLOGÍA}

La metodología en esta investigación fue la Investigación Aplicada. Está utiliza los conocimientos obtenidos en investigaciones previas, y con ello concientizar a la sociedad, contrastando los escenarios y el comportamientos de las variables objeto a estudios se basa en conocimiento científico de los hechos y del entorno. En el análisis de la contaminación lumínica en el medio ambiente, ecosistema y sus efectos en la población fue necesario profundizar en la misma.

\section{Lo Cotidiano}

El humano se mueve en un ambiente diario sin entender casi nada acerca del mundo. Dedicamos poco tiempo a pensar en el mecanismo que genera la luz solar que hace posible la vida, en la gravedad que nos ata a la Tierra y que de otra forma nos lanzaría al espacio, o en los átomos de los que estamos constituidos y de cuya estabilidad dependemos de manera fundamental.

Durante millones de años, los seres vivientes de la Tierra han ido adaptando sus procesos biológicos de acuerdo con dos ciclos astronómicos fundamentales: la sucesión de las estaciones y la alternancia día-noche. Dado que la percepción de ambos fenómenos es desigual según la latitud, las distintas especies se han acomodado a la singularidad de ambos ciclos en su hábitat. Cualquier perturbación en alguno de ellos originaría distorsiones cuyo alcance desconocemos, pero que, con toda seguridad, ocasionarían la extinción de algunas especies y la aparición de nuevas exigencias adaptativas para las demás. 


\section{Definición}

La contaminación lumínica (Light Pollution) podemos definirla como el efecto producido por la luz que no se aprovecha con la finalidad de iluminar distintas zonas de la ciudad como calles, edificios, rutas, carteles, etc. y se difunde en la atmósfera, generando una iluminación deficiente y un gasto energético inútil. Si las instalaciones lumínicas no están bien diseñadas o no son adecuadamente instaladas pueden resultar costosas e ineficientes.

La acción del hombre y su cultura sobre el medio ambiente está, en la actualidad, generando una seria alteración en ambos ciclos cósmicos. La actividad industrial y las formas de vida propias de la sociedad actual, de mantenerse el nivel de consumo energético. Niveles más elevados de "bienestar" exigen consumir cada vez más energía, actualmente, un ciudadano de un país industrializado consume 100 veces más energía que un habitante de un país no industrializado.

El consumo responsable de energía debería ser algo consubstancial a la educación cívica de la población por dos motivos. El primero de ellos: porque el actual modelo de consumo energético se basa en la conversión en energía de recursos naturales no renovables (carbón, petróleo y/o uranio), el segundo porque en los procesos de conversión en energía, transporte y su posterior consumo, se generan residuos que contaminan gravemente el medio ambiente.

Si bien la contaminación atmosférica por el $\mathrm{CO} 2$ emitido, amenaza con alterar el equilibrio climático (efecto invernadero por emisión de $\mathrm{CO} 2$ ). En la actualidad, el calentamiento global del planeta debido a este efecto es ya una evidencia científica y sus efectos devastadores sobre el clima son crecientes: lluvias torrenciales, huracanes catastróficos, inundaciones, sequías prolongadas, deshielo de los casquetes polares etc.

\section{IMPACTO SOBRE EL MEDIO NATURAL}

En las últimas décadas, el grado y la intensidad de la iluminación artificial de la noche se han incrementado de tal forma que hoy nadie puede negar sus efectos negativos sobre hábitats y especies. En relación a las áreas naturales y espacios protegidos han de distinguirse dos acepciones relativas a los efectos de la contaminación lumínica. Una es la "contaminación lumínica astronómica", es decir, la que altera la vista del cielo nocturno considerado como paisaje, y la otra acepción, se corresponde con la "contaminación lumínica ecológica", referida a la alteración de los regímenes de luminosidad naturales en los ecosistemas terrestres y acuáticos.

La progresiva ocupación del territorio provoca que los hábitats, ecosistemas y comunidades sensibles, se encuentren expuestos al impacto de la luz artificial en la noche, así como a los efectos directos de la contaminación atmosférica que reducen la calidad del cielo nocturno. Nuestro conocimiento actual de la gama completa de consecuencias ecológicas derivadas de la pérdida de calidad del cielo nocturno es todavía muy limitado. Se hace necesario tomar una mayor conciencia y responsabilidad de la necesidad de profundizar en la investigación y el desarrollo de metodología científica capaz de evaluar el alcance de estos fenómenos. Actualmente se tiene abundante información sobre los efectos causados en determinadas especies migratorias que se guían por 
Figura 2: Vista de la Tierra desde la Estación especial internacional

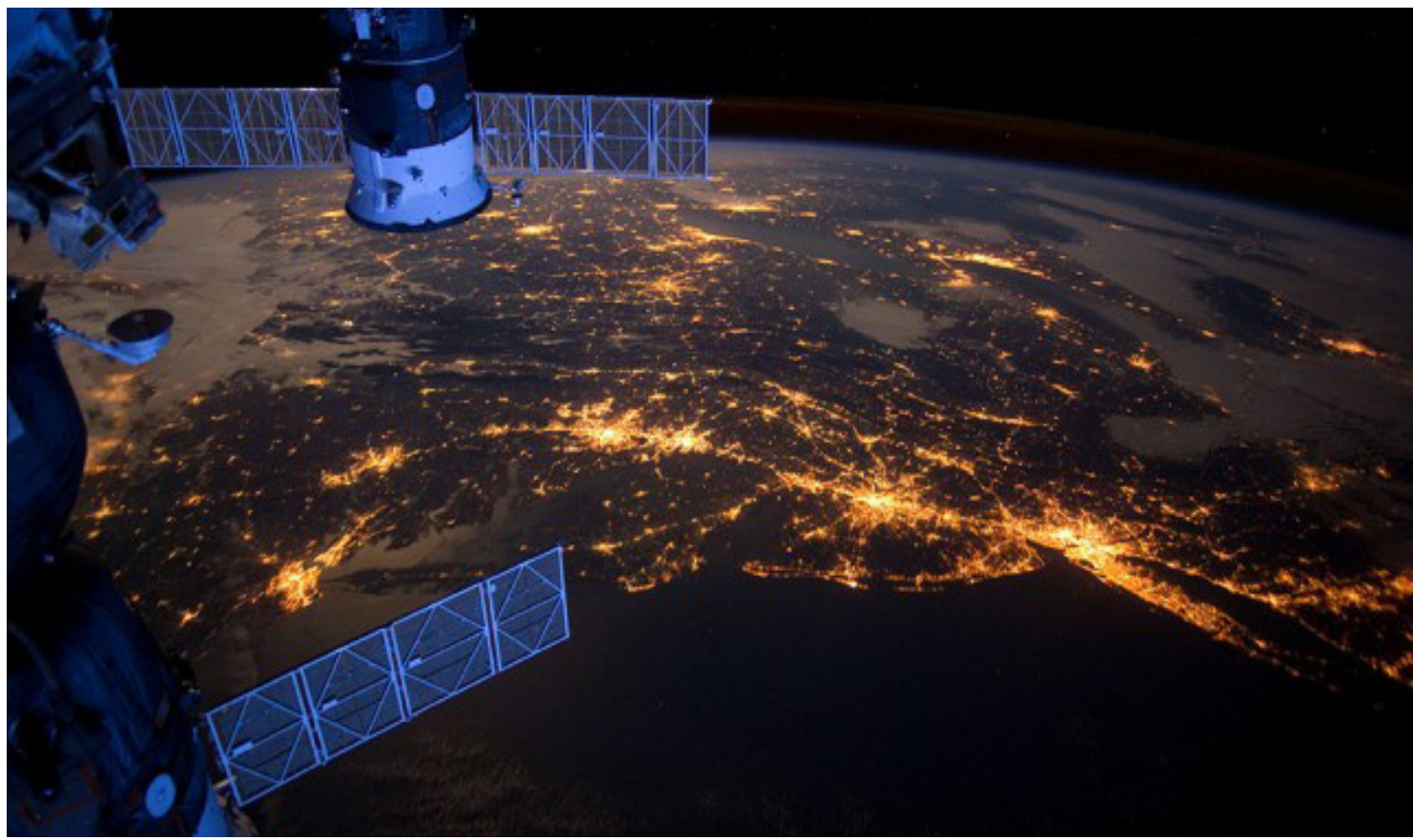

Fuente: National Aeronautics And Space Administration (NASA)

la luz de las estrellas, o sobre fenómenos tan evidentes como la muerte masiva por deshidratación de determinadas tortugas marinas desorientadas por las luces en sus playas natales.

Pero la extensión de la noche artificial en el medio natural provoca otros impactos no tan conocidos o evidentes. Entre ellos destaca la alteración de los ciclos de ascenso y descenso del plancton marino, lo que afecta a la alimentación de las especies marinas, o las incidencias desfavorables sobre el equilibrio poblacional de muchas especies, resaltando ámbitos como la perturbación de la numerosísima fauna de insectos nocturnos y el equilibrio depredador-presa. Preservar íntegramente la naturaleza implica conservar los "Paisajes de Luz Natural", especialmente en la noche.

Un "Paisaje de Luz Natural” es un entorno caracterizado por la influencia lumínica natural del sol y los ciclos lunares, por disponer de aire limpio, y de cielos oscuros no perturbados por la luz artificial. La inclusión de los cielos oscuros como dimensión en la protección de los paisajes de la noche, no representa solamente un recurso esencial en su mantenimiento o preservación, sino que también contribuye a enriquecer las múltiples experiencias de los visitantes y abrir nuevos escenarios para las ciencias naturales. 
Por otro lado un estudio realizado por científicos de la Administración Nacional Oceánica y Atmosférica (NOAA) y de la Universidad de Colorado, indica que el exceso de luz durante la noche debido a la polución lumínica contribuye a la contaminación del aire. Todas las noches, los químicos y gases tóxicos producidos por los vehículos y otras industrias son descompuestos por el óxido de nitrógeno, evitando que se transformen en smog, ozono u otros gases irritantes. Este compuesto químico sólo funciona durante la noche ya que es destruido por la luz del sol.

A pesar de que la luz que emana una metrópolis es 10.000 veces más tenue que la del sol, los estudios demuestran que las luces urbanas suprimen esta molécula. "Nuestros primeros resultados indican que las luces de la ciudad pueden hacer más lento el proceso de limpieza nocturno en hasta un $7 \%$ y también pueden aumentar los productos químicos iniciales para la contaminación del ozono, al día siguiente, en hasta un 5\%", Harald Stark, de (NOAA).

Figura 3 : comportamiento y proyección de Light Pollution EEUU



Fuente google earth. 2015 
Los espacios naturales protegidos deben ser por propia definición, ámbitos preferentes del desarrollo de iniciativas de protección de la luz natural de la noche. En particular se destaca el papel de laboratorios de la conservación de la noche que pueden jugar espacios emblemáticos como los incluidos en la Red Mundial de Reservas de Biosfera, los Parques Nacionales, los sitios Patrimonio de la Humanidad o los lugares incluidos en la lista de Ramsar ${ }^{1}$, capaces de generar nuevos conocimientos científicos y sistemas de gestión avanzada sobre los factores relacionados con la luz que inciden en el periodo nocturno respecto a la conservación de la biodiversidad.

La calidad astronómica de un observatorio está principalmente definida por la transparencia de sus cielos y por el número de horas de observación útil al año. Esto está íntimamente relacionado con la climatología del lugar y de sus características geográficas, así como por la ausencia de factores adversos que dificulten la observación. Los requerimientos de calidad del cielo en la observación astronómica reducen sustancialmente los lugares en el planeta que pueden considerarse apropiados, por lo que inevitablemente, tales ámbitos han de considerarse como un recurso escaso que es necesario preservar.

\section{EFECTOS DE LA CONTAMINACIÓN LUMÍNICA SOBRE LA SALUD HUMANA}

La utilización de las fuentes de luz artificial tras la puesta del sol ha permitido al hombre llevar a cabo tareas y gozar de diversas actividades durante mucho más tiempo. De hecho, la luz durante la noche se ha convertido en algo tan común que en muchos lugares del mundo la verdadera oscuridad está virtualmente desapareciendo. Los científicos biomédicos se han dado cuenta de que la exposición a la luz durante la noche también tiene consecuencias para la fisiología humana y animal.

La luz nocturna, cuando es de suficiente intensidad y de la longitud de onda apropiada (azul), es convertida a una señal eléctrica que viaja al sistema nervioso central. Esta señal altera la función del reloj biológico y en última instancia, la producción de melatonina en la glándula pineal, que segrega fundamentalmente de forma exclusiva durante la noche, presenta una serie de importantes funciones que se pierden en el momento en el que existe exposición nocturna a la luz. Algunas de estas acciones incluyen la modulación del ciclo sueño/vigilia, la regulación de los ritmos circadianos, y su sincronización.

Así, la reducción de la melatonina por luz durante la noche informa a muchos de nuestros órganos que es de día, cuando, de hecho, es de noche, por lo que reajustan su fisiología en concordancia. Esta información desvirtuada

1 Convención Relativa a los Humedales de Importancia Internacional especialmente como Hábitat de Aves Acuáticas, conocida en forma abreviada como Convenio de Ramsar, fue firmada en la ciudad de Ramsar (Irán) el 18 de enero de 1971 y entró en vigor el 21 de diciembre de 1975. Su principal objetivo es: «la conservación y el uso racional de los humedales mediante acciones locales, regionales y nacionales y gracias a la cooperación internacional, como contribución al logro de un desarrollo sostenible en todo el mundo». 
puede tener consecuencias más graves que simplemente una leve sensación de malestar. El sistema circadiano² de los mamíferos se compone de una red de estructuras, organizada jerárquicamente, responsable de la generación y sincronización de los ritmos circadianos con el medio ambiente.

Esta red de estructuras está integrada por un marcapasos central, situado en el núcleo supraquiasmático ${ }^{3}$ (NSQ) del hipotálamo, y varios osciladores periféricos. El NSQ transmite señales temporales rítmicas a todos los órganos y tejidos a través de mediadores humorales (entre los cuales la hormona melatonina juega un papel principal) y del sistema nervioso autónomo.

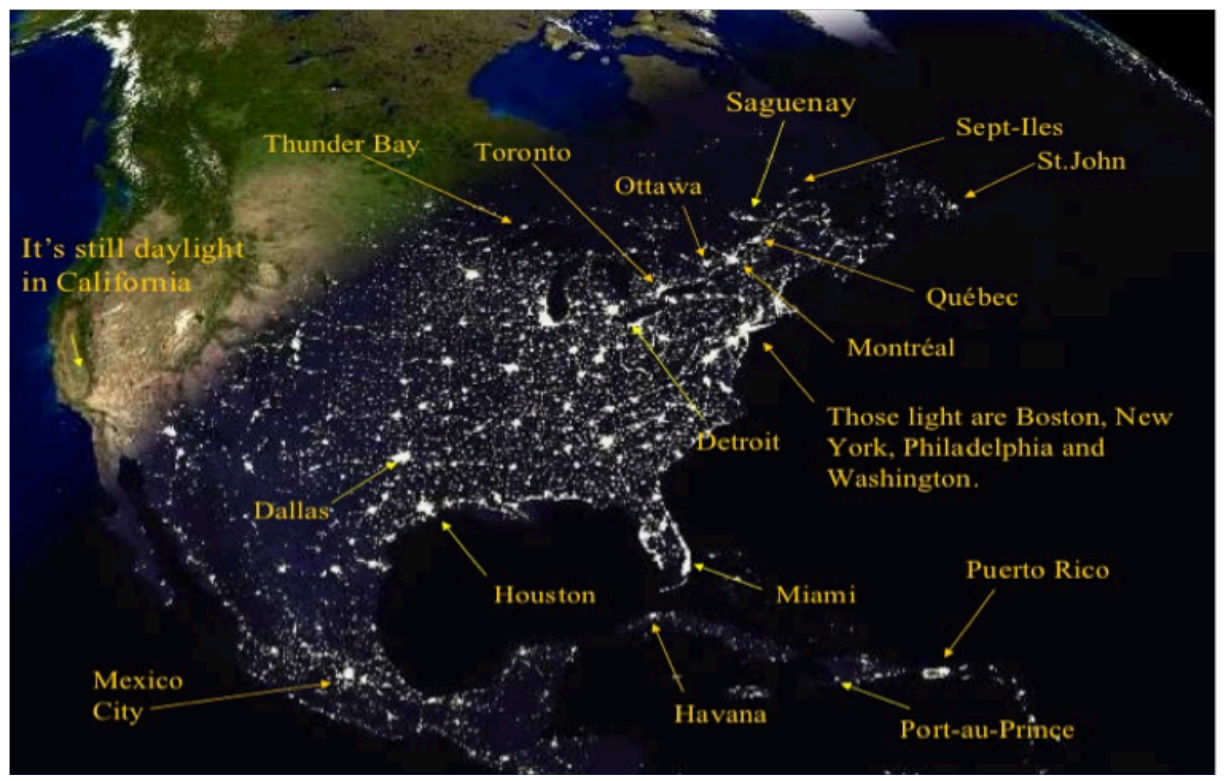

Figura 4: google earth. 2015

2 Los seres vivos presentan una clara organización temporal en su conducta y los procesos fisiológicos bioquímicos y morfológicos subyacentes, que se manifiesta en los ritmos biológicos. Estos se denominan ritmos circadianos cuando persisten con un periodo cercano a las $24 \mathrm{~h}$ en condiciones libres de señales ambientales de tiempo. ultradianos (periodo con periodo menor de 24 horas) e infradianos (periodo mayor de 24 horas) (Cardinali y cols., 1994).

3 En los mamíferos, el núcleo supraquiasmático es una estructura bilateral que se localiza en la porción ventral del hipotálamo anterior, ventrolateral al receso óptico del tercer ventrículo y dorsal al quiasma óptico. Las neuronas del NSQ expresan diversas sustancias neuroactivas, las neuronas del NSQ sincronizan su actividad con el ciclo día-noche. La cantidad de luz ambiental es detectada en la retina por fotorreceptores circadianos diferentes a los que intervienen en la visión. 
Entre todas las señales ambientales, la alternancia de luz-oscuridad cada 24 horas es el sincronizador más importante del NSQ. En condiciones naturales, el NSQ se reajusta todos los días gracias a la señal luminosa que le llega a través de una vía no visual que se inicia en un subgrupo de células ganglionares de la retina que poseen un fotopigmento ${ }^{4}$, la melanopsina, que es particularmente sensible a la luz azul.

La insuficiente exposición a luz diurna y/o la excesiva exposición a luz brillante por la noche perjudican el funcionamiento del NSQ, afectando a los ritmos de cortisol y melatonina, dos de las señales humorales más importantes que transmiten la señal luminosa a los tejidos periféricos. En las sociedades modernas se están implantando jornadas de trabajo continuadas de 24 horas, hecho que se traduce en un aumento de la proporción de población que participa habitualmente en trabajo por turnos y que, en consecuencia, sufre disfunción circadiana o cronodisrupción ${ }^{5}$; es decir, pérdida del orden temporal interno: los ritmos fisiológicos dejan de estar coordinados entre sí.

Una de las salidas del NSQ mejor conocidas es la vía multisináptica ${ }^{6}$ que alcanza la glándula pineal, responsable de la síntesis de melatonina, hormona que difunde el mensaje temporal del NSQ al resto del organismo, su síntesis está sometida a una doble regulación: por un lado la estimulación noradrenérgica ${ }^{7}$, por parte del NSQ que tiene lugar durante la noche y por otro, a la acción directa inhibidora de la luz. Así, la producción de esta hormona muestra un marcado ritmo circadiano, caracterizado por bajos valores durante el día y elevados durante la noche, con independencia del carácter nocturno o diurno de los organismos, lo que ha llevado a que se la conozca como la "oscuridad química".

Los cambios fisiológicos ocasionados por la contaminación lumínica, pueden a largo plazo, traducirse en procesos fisiopatológicos que contribuirían a la enfermedad. Así, por ejemplo, se ha detectado un aumento del riesgo de padecer cáncer en individuos expuestos de forma repetida y/o prolongada a luz excesiva, como

4 Los fotorreceptores circadianos son un tipo especial de célula ganglionar que poseen el fotopigmento melanopsina, y que se caracterizan por tener un campo receptivo visual muy amplio y codificar la cantidad de luz

5 Fenómeno relativamente nuevo, denominado cronodisrupción (CD) que puede conducir a un envejecimiento prematuro. La CD se ha definido como una alteración relevante del funcionamiento del sistema circadiano; es decir, del orden temporal interno de los ritmos circadianos bioquímicos, fisiológicos y de comportamiento. Se considera también como la ruptura de la relación de fase normal entre los ritmos circadianos internos y los ciclos de 24-h del medio ambiente.

6 Prolongación nerviosa o al sistema de células nerviosas que requieren múltiples sinapsis.

7 Neuronas que utilizan la noradrenalina como neurotransmisor. La mayoría de los cuerpos celulares de las neuronas noradrenérgicas se localizan en el tronco cerebral, en una zona llamada locus coeruleus.

8 La oscuridad química, se denomina al proceso de sintetización de la melatonina por la glándula pineal en el ciclo luz-oscuridad. 
ocurre en los empleos del turno de noche. Debido a las múltiples acciones de la melatonina como neutralizadora de radicales libres y antioxidante de amplio espectro, privar al cuerpo de esta importante sustancia puede contribuir a la iniciación, progresión o gravedad de toda una serie de enfermedades.

Especialmente en ancianos, incluyen como parte de su proceso degenerativo la acumulación de daño oxidativo por radicales libres. Debido a su eficacia para privar al cuerpo de la melatonina pineal, está claro que debemos evitar la exposición a la luz durante la noche siempre que sea posible. Desafortunadamente, evitar la luz nocturna es poco factible en el mundo actual. Por lo tanto, algunas alternativas más prácticas serían el desarrollo de fuentes lumínicas que excluyan las longitudes de onda específicas que inhiben la síntesis de melatonina, la producción de gafas o lentes de contacto que filtren estas longitudes de onda y la fabricación de pantallas para lámparas que reduzcan la luz que las traspasa.

El mensaje fundamental es que cualquier factor que limite la producción circadiana endógena de melatonina, como por ejemplo la exposición nocturna a la luz de consecuencias, en cuanto a enfermedades y al proceso del envejecimiento. El abuso de la luz no debe pasarse por alto puesto que potencialmente contribuiría a la enfermedad en individuos jóvenes y aumentaría la debilidad y la enfermedad en los ancianos.

\section{Resultados. ¿Qué hacer?}

Hay que evitar la emisión directa de luz hacia el cielo, cosa que se consigue usando luminarias orientadas en paralelo al horizonte, con bombillas bien apantalladas y eficientes, con potencia necesaria sin sobre dimen-

Figura 6: Espectro lumínico de las lámparas de alumbrado público.

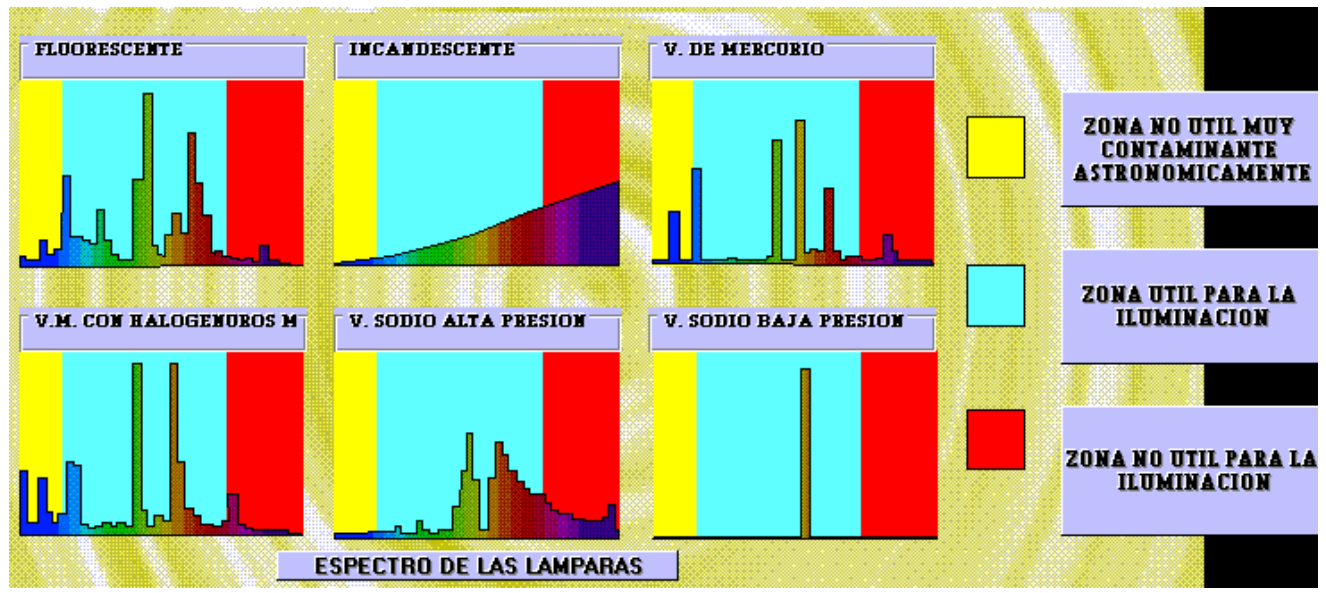

Fuente. Instituto Ecuatoriano de Normalización, (INEN) Reglamento técnico Ecuatoriano "RTE INEN 069 Alumbrado Público" 
sionamiento tanto de potencia (watt) para alumbrar el suelo de acuerdo con los criterios de seguridad, pero no más. También, es aconsejable emplear con preferencia las luminarias que tengan el vidrio refractor de cerramiento plano y transparente.

La zona del medio (celeste) es la zona útil para iluminación, pues es la región en la cual el ojo humano es sensible. No tiene sentido utilizar lámparas que emitan en el espectro ultravioleta ni en el infrarrojo pues consumirán energía, no iluminarán y dicha emisión se dispersará en la atmósfera. Si bien no será detectada por el ojo, sí lo será por algunos otros seres vivos y por los diferentes tipos de detectores que se utilizan en la observación astronómica.

Las lámparas de vapor de mercurio ofrecen además el peligro de contaminación química pues al romperse, el vapor de mercurio pasa a la atmósfera. Las lámparas de vapor de sodio a baja presión son las más adecuadas pues emiten en una estrecha región del espectro electromagnético en la cual el ojo es sensible (toda la emisión se aprovecha) a la vez que sus efectos en el cielo son más fáciles eliminar.

Los astrónomos lo harían colocando un filtro de banda angosta en los detectores de uso astronómico y el ciudadano común interesado en observar objetos más débiles en el cielo, simplemente tendría que colocarse

Figura 7: Normas básicas de utilización del alumbrado

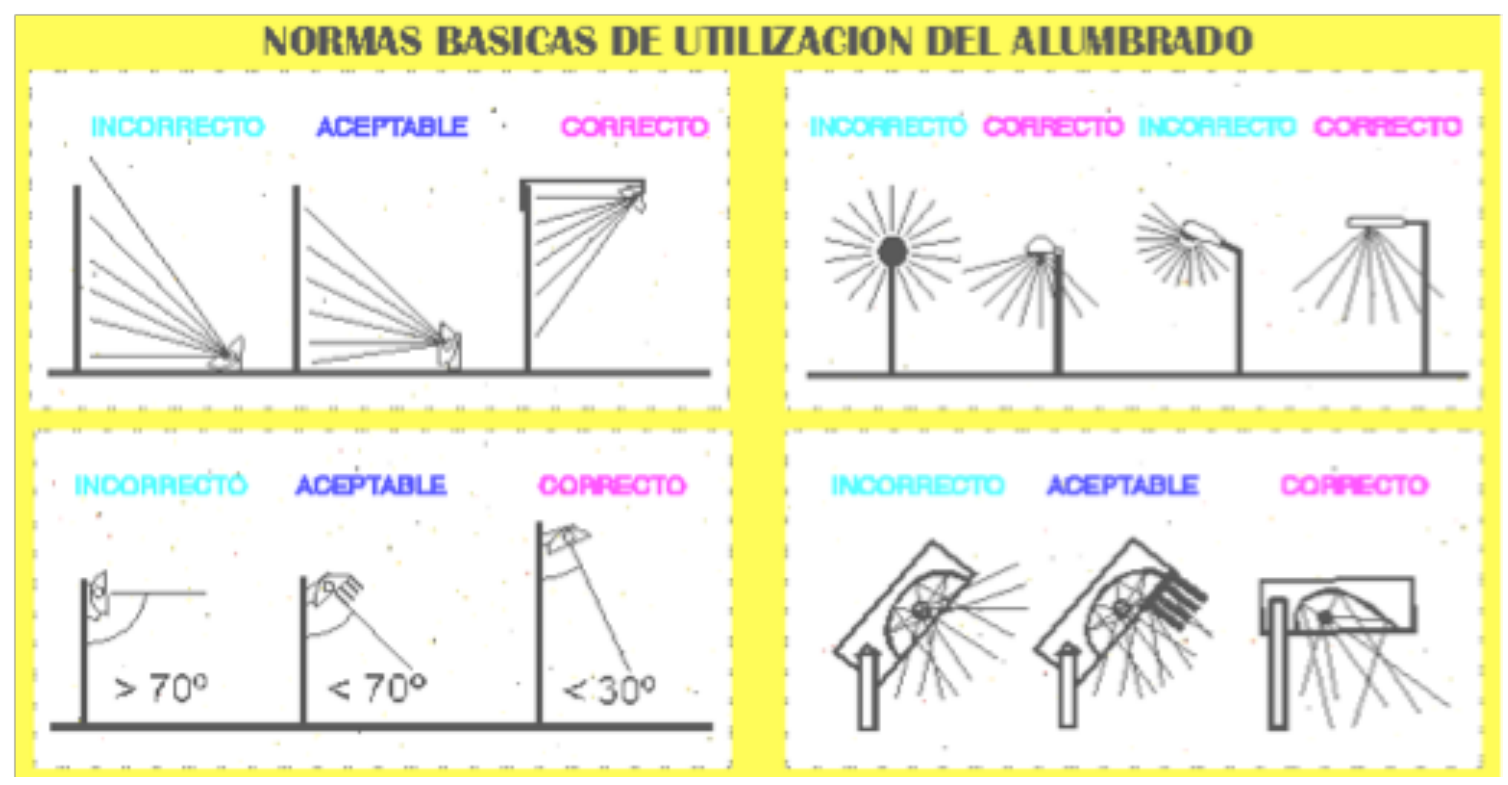

Fuente: http://editorial.cda.ulpgc.es/instalacion/3_ALUMBRADO/36_contaminacionA/lumi6.gif google. 2015 
anteojos con el mismo filtro. Estos filtros ya existen y son conocidos como "filtros anti-polución", utilizados fundamentalmente en fotografía nocturna.

El diseño del artefacto que alberga la lámpara así como su colocación también debe ser cuidadosamente planificado según el fin buscado. Especialmente los utilizados en alumbrado público deben ser tipo (cut-off), es decir, con cero emisión en la dirección del hemisferio superior.

Añadir el apagado de alumbrados ornamentales y de grandes espacios exteriores que resultan injustificables a partir de cierta hora, los cuales pueden alumbrarse con potentes proyectores orientados incorrectamente que dispersan mucha luz hacia el cielo y también en direcciones laterales. Si esto se hace, se aprovecha al máximo la energía y se reduce considerablemente el consumo. También hay que remodelar este tipo de alumbrado, cambiando bombillas, variando su inclinación y utilizando dispositivos que eviten la dispersión de la luz fuera del área a iluminar.

Figura 8: Así se verían Hong Kong y New York en la noche sin la contaminación lumínica.
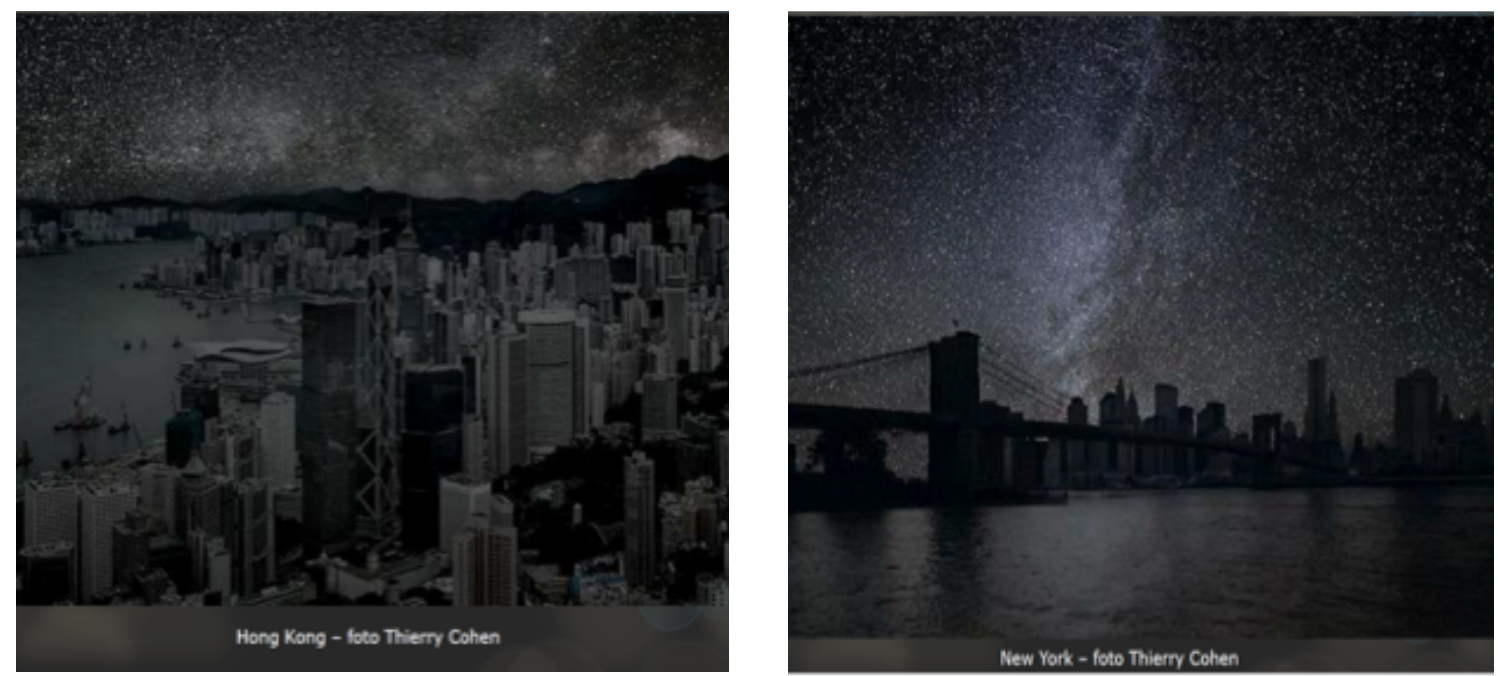

Fuente: Google, fotos tomadas por Thierry Cohen, 2015 


\section{CONCLUSIONES}

La observación desde un satélite del cielo nocturno nos muestra a una escala global el desarrollo de las naciones, resaltando los países desarrollados como Estados Unidos, Europa y Japón y sus grandes ciudades las severas condiciones de la Light Pollution donde el cielo nocturno ya no es observable como en tiempo de antaño todas estas miles de personas que habitan estas ciudades están expuestas a los cambios del ritmos circadianos, originando y/o perjudicando el funcionamiento del NSQ, afectando a los ritmos de cortisol y melatonina, dos de las señales humorales más importantes que transmiten la señal luminosa a los tejidos periféricos.

Estos cambios fisiológicos ocasionados por la contaminación lumínica, pueden a largo plazo, traducirse en procesos fisiopatológicos que contribuirían a enfermedades. La cual afectará a un número considerable de la población mundial, por tal motivo debemos tomar conciencia y unir esfuerzo en la promulgación de legislaciones donde se considere tal situación en la mayoría de las naciones y que vaya de la mano al desarrollo urbano e industrial de nuestra sociedad.

"Las Personas de las generaciones futuras tienen derecho a una Tierra indemne y no contaminada, incluyendo el derecho a un cielo puro".

Declaración universal de los derechos de las generaciones futuras de 1997, UNESCO.

\section{Legislaciones al Respecto}

- Estado de Nueva York Legislación aprobada en jurisdicciones de condados y ciudades. Proyecto de Ley A01182B aprobado (104-39) en la legislatura estatal reconoce aspectos astronómicos, económicos, ecológicos, seguridad y de salud pública

- Chile. Aprobación de Norma de Emisión para la Regulación de la Contaminación Lumínica D.S. No. 686/98 del Ministerio de Economía Fomento y Reconstrucción

- Puerto Rico 2008 - 1ro por consumo energético por km2 Aprobación Ley 248 de 2008 Reconoce aspectos ambientales, energéticos y astronómicos.

- Resolución XXIII Asamblea General en Kyoto, Agosto de 1997 (http://www.aas.org/ light/pollution_iau_resolution_97.html)

- Resolución XX Asamblea General de la UAI

"strongly urges (a) that all astronomers request civil authorities and others in their countries to implement solutions to preserve the quality of observing conditions, and (b) that all national organizations bring these concerns to the notice of adhering organizations, space agencies, and others in their countries" (IAU Today No. 10, 1988 Aug. 11). 


\section{- $\quad$ PROTECTION OF THE NIGHT SKY}

The XXIII International Astronomical Union General Assembly, Considering that Proposals have been made repeatedly to place luminous objects in orbit around the earth to carry messages of various kind and that the implementation of such proposals would have deleterious effects on astronomical observations, and that the night sky is the heritage of all humanity, which should therefore be preserved untouched, Requests the President to take steps with the appropriate authorities to ensure that the night sky receive no less protection than has been given to the world heritage sites on earth. 


\section{BIBLIOGRAFÍA}

CINZANO, P. 1997. Inquinamento luminoso e protezione del cielo Notturno. Memorie, Classe di Scienze Fisiche, Matematiche e Naturali, Volume XXXVIII, Istituto Veneto di Scienze, Lettere ed Arte, Venezia http://www.lightpollution.it/cinzano/libro/index.html

CINZANO, P. 2002. Technical measures for an effective limitation of the effects of light pollution. En CINZANO, P. (coord.), Light Pollution and the Protection of the Night Environment, Thiene (Italia), 2002 http://www.lightpollution.it/istil/venice/index.html

Universidad Nacional de San Juan EL VALOR DEL RECURSO CIELO - PAISAJE NOCTURNO Y LA CONTAMINACION LUMINICA Yanina N. Buchiniz1, María Gabriela Torre1, Hector S. Lepez2 1 FATU UNCo (Neuquén - Argentina , 2 OAFA - FCEFN - UNSJ.yanina_buchiniz@yahoo.com.ar

HOLLAND, J. 2008. What is light pollution, and how do we quantify it? http://amper.ped.muni.cz/light/lp_what_is.pdf REGLAMENTO TÉCNICO ECUATORIANO RTE INEN 069 “Alumbrado Público”

La Unión Astronómica Internacional (UAI)

Ley catalana para la protección del medio nocturno

Sociedad Española de Astronomía

Departamento de Astrofísica y Ciencias de la Atmósfera de la Universidad Complutense de Madrid

National Aeronautics And Space Administration (NASA), Last Updated: Aug. 11, 2015

US NATIONAL Library of medicine National institutes of Health, Rajaratnam SM, < Arendt J. Health in a 24h-society. Lancet. 2001

US NATIONAL Library of medicine National institutes of Health, Stevens RG. <

Circadian disruption and breast cancer: from melatonin to clock genes. Epidemiology.2005.

US NATIONAL Library of medicine National institutes of Health, Reiter RJ, Tan DX, Korkmaz A, Erren TC, Piekarski C, Tamura H, et al. Light at night, chronodisruption, melatonin suppression, and cancer risk: a review. Crit Rev Oncog.2007.

US NATIONAL Library of medicine National institutes of Health, Craig LA, McDonald RJ. Chronic disruption of circadian rhythms impairs hippocampal memory in the rat. Brain Res Bull. 2008.

US NATIONAL Library of medicine National institutes of Health, Erren TC, Reiter RJ, Piekarski C. Light, timing of biological rhythms, and chronodisruption in man. Naturwissenschaften. 2003. 
US NATIONAL Library of medicine National institutes of Health, Rahman SA, Kollara A, Brown TJ, Casper RF. Selectively filtering short wavelengths attenuates the disruptive effects of nocturnal light on endocrine and molecular circadian phase markers in rats. Endocrinology. 2008. 